\title{
CME Motor signs predict poor outcomes in Alzheimer disease
}

\author{
N. Scarmeas, MD; M. Albert, PhD; J. Brandt, PhD; D. Blacker, MD, ScD; G. Hadjigeorgiou, MD; \\ A. Papadimitriou, MD; B. Dubois, MD; M. Sarazin, MD; D. Wegesin, PhD; K. Marder, MD, MPH; \\ K. Bell, MD; L. Honig, MD, PhD; and Y. Stern, PhD
}

\begin{abstract}
Objective: To examine whether the presence of motor signs has predictive value for important outcomes in Alzheimer disease (AD). Methods: A total of 533 patients with AD at early stages (mean Folstein Mini-Mental State Examination [MMSE] 21/30 at entry) were recruited and followed semiannually for up to 13.1 years (mean 3) in five University-based $\mathrm{AD}$ centers in the United States and European Union. Four outcomes, assessed every 6 months, were used in Cox models: cognitive endpoint (Columbia Mini-Mental State Examination $\leq 20 / 57$ [ MMSE $\leq 10 / 30]$ ), functional endpoint (Blessed Dementia Rating Scale $\geq 10$ ), institutionalization equivalent index, and death. Using a standardized portion of the Unified PD Rating Scale (administered every 6 months for a total of 3,149 visit-assessments, average 5.9 per patient), the presence of motor signs, as well as of individual motor sign domains, was examined as time-dependent predictor. The models controlled for cohort, recruitment center, sex, age, education, a comorbidity index, and baseline cognitive and functional performance. Results: A total of 39\% of the patients reached the cognitive, $41 \%$ the functional, $54 \%$ the institutionalization, and $47 \%$ the mortality endpoint. Motor signs were noted for $14 \%$ of patients at baseline and for $45 \%$ at any evaluation. Their presence was associated with increased risk for cognitive decline (RR, 1.72; 95\% CI, 1.24 to 2.38), functional decline (1.80 [1.33 to 2.45]), institutionalization (1.68 [1.26 to 2.25]), and death (1.38 [1.05 to 1.82$]$ ). Tremor was associated with increased risk for reaching the cognitive and bradykinesia for reaching the functional endpoints. Postural-gait abnormalities carried increased risk for institutionalization and mortality. Faster rates of motor sign accumulation were associated with increased risk for all outcomes. Conclusions: Motor signs predict cognitive and functional decline, institutionalization, and mortality in Alzheimer disease. Different motor sign domains predict different outcomes.
\end{abstract}

NEUROLOGY 2005;64:1696-1703

Motor signs are commonly observed in Alzheimer disease $(\mathrm{AD}){ }^{1-4}$ Because their exact mechanism and anatomic location of origin is not clear, in this article we use the term motor signs to refer to what is usually described in the literature as extrapyramidal signs or parkinsonian signs or parkinsonism.

As compared to patients with $\mathrm{AD}$ without motor signs, patients with $\mathrm{AD}$ with motor signs have higher annual total cost of care. ${ }^{5}$ In addition, information about presence of motor signs in $\mathrm{AD}$ is important because they may predict cognitive $^{6-9}$ and functional ${ }^{2,10}$ decline, institutionalization, ${ }^{2,10}$ and death. ${ }^{10-16}$ However, some reports failed to detect significant associations for cognition, ${ }^{2,17-19}$ function, ${ }^{17,18,20}$ institutionalization, ${ }^{20}$ or mortality. ${ }^{21,22}$

Many factors contribute to the variability in re-

Additional material related to this article can be found on the Neurology Web site. Go to www.neurology.org and scroll down the Table of Contents for the May 24 issue to find the title link for this article. ported associations between motor signs and $\mathrm{AD}$ outcomes. Some of the inconsistency derives from variability in the definitions of motor signs, inconsistent consideration of treatments with neuroleptics, use of standardized scales vs clinical evaluation, small sample size resulting in limited power, inclusion of subjects at varying stages of disease, and variable levels of participation at and duration of follow-up. Also, many studies considered motor signs globally, and only a few reports have focused on individual domains of motor signs. In addition, most previous studies considered motor signs only at a single point during the course of $\mathrm{AD}$, typically at the baseline visit or less frequently at any point during the disease course. Because of the progressive nature of $\mathrm{AD}$, motor signs are not static and invariable but may fluctuate from visit to visit. ${ }^{3,4}$ Therefore, consideration of motor signs as fixed predictors may lead to biased results.

To obtain a more definitive answer to these ques-

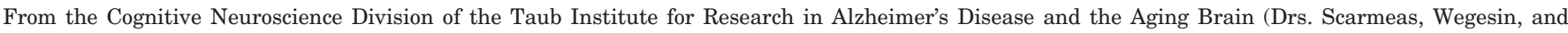
Stern), the Gertrude H. Sergievsky Center (Drs. Scarmeas, Marder, Bell, Honig, and Stern) and the Department of Neurology (Drs. Scarmeas, Marder, Bell,

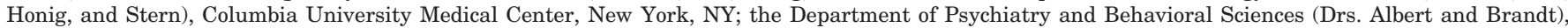
Johns Hopkins University, Baltimore, MD; the Department of Psychiatry (Dr. Blacker), Massachusetts General Hospital, Harvard Medical School, Boston; the Department of Neurology (Drs. Hadjigeorgiou and Papadimitriou), University of Thessaly, Larissa, Greece; and the Department of Neurology (Drs. Dubois and Sarazin), Hospital de la Salpetriere, Paris, France.

Supported by Federal grants AG07370, RR00645, P50-AG 08702, and the Taub Institute for Research on Alzheimer's Disease and the Aging Brain.

Received October 19, 2004. Accepted in final form January 31, 2005.

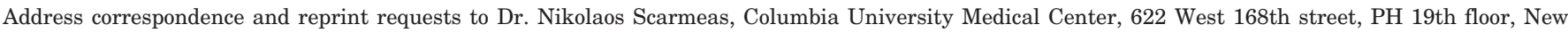
York, NY 10032; e-mail: ns257@columbia.edu

1696 Copyright (C) 2005 by AAN Enterprises, Inc 
tions, we analyzed data from the Predictors Study, a large, multicenter study of patients with probable $\mathrm{AD}$ followed from the early stages of the disease for up to 13 years. Standardized assessments of motor signs were administered semiannually. We assessed their association with four outcomes: cognitive endpoint, functional endpoint, institutionalization, and death. Taking advantage of the multiple assessments of motor signs throughout the course of the disease, we were able to consider their predictive ability in a time-dependent fashion.

Methods. Participants. Subjects from two Predictors Study cohorts $^{23}$ were included in these analyses. For the Predictors 1 cohort, patients were recruited and studied at three sites in the United States: Columbia University, New York; Johns Hopkins University, Baltimore; and Harvard University, Boston. For the Predictors 2 cohort, two additional sites in the European Union were added: Hospital de la Salpetriere, Paris, France; and University of Thessaly, Larissa, Greece. The study was approved by the appropriate local Institutional Review Boards.

The inclusion and exclusion criteria, as well as the evaluation procedures of the Predictors Study, have been described in more detail elsewhere. ${ }^{23,24}$ Briefly, patients met Diagnostic and Statistical Manual of Mental Disorders (DSM-III-R) criteria for primary degenerative dementia of the Alzheimer type and National Institute of Neurologic Disorders and Stroke-AD and Related Disorders Association criteria for probable AD. Enrollment required a Columbia Mini-Mental State Examination (CMMS) score of 30 or more (maximum CMMS score, 57), which is equivalent to a score of approximately 16 or more on the Folstein Mini-Mental State Examination (MMSE). ${ }^{25,26}$ There were 42 patients who were recruited despite having CMMS score of $<30$ at baseline. Because their exclusion did not change the results, we included them in the present analyses. Exclusion criteria were diagnosis of PD or parkinsonism at any time prior to the onset of dementia, history of alcohol abuse or dependence, any electroconvulsive treatment within 2 years of recruitment or 10 or more electroconvulsive sessions at any time, history or current clinical evidence of schizophrenia or schizoaffective disorder that started before the onset of intellectual decline, and evidence of stroke from either medical history or clinical examination or a Hachinski Ischemic Score of $\geq$ 5 or strokes in cortical areas or focal atrophy resulting from focal infarcts in either CT or MRI. Periventricular or subcortical abnormal CT or MRI signals consistent with small vessel ischemic changes and small lacunes were permitted. Each consecutive patient who met these study criteria was included, except for those who did not consent to participate or who lived too far away and were unable to return to the hospital for regular follow-up.

During follow-up, in every bi-annual assessment the occurrence of a stoke during the last 6 months was recorded. Overall 15 patients developed a stroke at some point during the follow-up. Because excluding them did not change the results they are considered in the presented analyses. Patients' clinical diagnoses were revisited yearly and only subjects who were consistently thought to have clinical $\mathrm{AD}$ were included in the analyses. Overall, 109 patients have come to autopsy examination. The postmortem diagnostic procedure has been completed for 96 patients, $96 \%$ of whom had $\mathrm{AD}$-type pathologic changes $(90 \%$ received the pathologic diagnosis of $\mathrm{AD}$ and $6 \%$ had senile changes of the Alzheimer type). Dementia with Lewy bodies was diagnosed in 23\% (coexisting with $\mathrm{AD}$-type changes in all but 1 patient). In the analyses presented in this article all subjects with clinical diagnosis of $\mathrm{AD}$ (irrespective of pathologic confirmation) were included. In secondary analyses, which excluded the few autopsied cases that did not have AD-type pathologic changes, the results were unchanged.

Evaluation. Predictors. At the initial visit, demographic characteristics (age, ethnicity, sex, education) were recorded and disease severity features were assessed.

Motor signs were assessed by trained examiners using a modified Unified Parkinson's Disease Rating Scale (UPDRS) that has good inter-rater reliability properties in dementia populations. ${ }^{27}$ More specifically, excellent agreement for all five rigidity items and gait and fair to good agreement for speech, facial expression, posture, and body bradykinesia-hypokinesia has been demonstrated for the use of this scale. ${ }^{27}$ Because no tremor was detected in our earlier study, adequate assessment of tremor reliability could not be performed, but good to excellent reliabilities for the UPDRS tremor items have been demonstrated in other studies. ${ }^{28}$

The following 11 items were rated on a scale of 0 to 4 (with 0 being normal and 4 indicating maximum impairment): speech, tremor at rest (in any limb), facial expression, neck rigidity, right arm rigidity, left arm rigidity, right leg rigidity, left leg rigidity, posture, gait, body bradykinesia-hypokinesia. For clinical purposes, the 11 items were grouped into the following five domains: Speech-Facial Expression (2 items, range 0 to 8), Tremor (1 item, range 0 to 4 ), Rigidity (5 items, range 0 to 20 ), Posture-Gait (2 items, range 0 to 8 ), and Bradykinesia ( 1 item, range 0 to 4 ). The grouping of individual UPDRS items into the five domains was guided by the following: 1) previous classifications that our group ${ }^{4,27,29}$ has used, 2) classifications used by other groups, ${ }^{2,3} 3$ ) factor analyses performed in multiple other cohorts ${ }^{30}$ and 4) a principal component analysis with varimax rotation-Kaiser normalization using data from the first evaluation of our patients, which produced factors broadly corresponding the above domains. A dichotomous motor sign variable was used: total score of $\geq 2$ vs total score of $<2$. The reason for this cutoff is as follows: 1 ) we previously demonstrated good interrater reliability for severity at least mild to moderate (i.e., score greater than 1 [which indicates slight severity]), ${ }^{27}$ and 2) this level of severity is also more likely to be noted by the average clinician. The domain subscores were also used in dichotomous forms (domain score of $\geq 2$ [if any of the items constituting the domain had a score $\geq 2$ ] vs domain score of $<2$ ). Total motor signs score (sum of all items) was included in the analyses in the form of continuous variable (range 0 to 44). The domain subscores were also utilized in a continuous form (sum of individual items of each domain). When interpreting the results we considered as most important only the associations noted with both the dichotomous and the continuous forms of motor sign domains.

Only non-drug induced motor signs were considered for these analyses. More specifically, at every 6-month visit the following two items were recorded: 1 ) Was the patient on medications that could cause motor signs (such as neuroleptics)? 2) Had the patient taken such medications in the past but did not continue to do so? If there was an affirmative answer to either of the two questions, the particular subject-evaluation was excluded from the analyses. In supplementary analyses we completely excluded all evaluations of subjects who ever had drug-induced motor signs and recomputed the models in the remaining 371 patients: the results were essentially unchanged.

A modified version of the Charlson Index of Comorbidity ${ }^{31}$ (referred to as comorbidity index) included items for myocardial infarct, congestive heart failure, peripheral vascular disease, hypertension, chronic obstructive pulmonary disease, arthritis, gastrointestinal disease, mild liver disease, diabetes, chronic renal disease, and systemic malignancy from initial visit. All items received weights of one, with the exception of chronic renal disease and systemic malignancy, which were weighted two. No patients with clinical strokes, metastatic tumors, or AIDS were included in the sample. At baseline visit $67 \%$ of patients had a comorbidity index of $0,19 \%$ an index of $1,9 \%$ an index of $2,4 \%$ an index of 3 , and $1 \%$ an index of 4 . Therefore, dichotomized scores $(0[67 \%]$ vs $\geq 1$ [33\%]) were used. Exploratory use of the index in a trichotomized ( 0 vs 1 vs $\geq 2$ ) or a continuous form did not change the results.

Outcomes. Cognitive outcome. Neurologic and mental status examinations were conducted at study entry and at 6-month intervals thereafter. The cognitive function measure used for the analysis was the CMMS ${ }^{25,26}$ (in English for the US sites and in French and Greek translated versions for the EU sites). This is a 57-point version of the original $\mathrm{MMSE}^{25}$ that includes the addition of digit span forward and backward, two additional calculation items, recall of the current and four previous political leaders of the country, confrontation naming of 10 items from the Boston Naming Test, one additional sentence to repeat, and a different copy task including two figures. We used a CMMS score of $\leq 20 / 57$ (equivalent to $\sim$ MMSE $\leq 10 / 30$ ) as the cognitive outcome endpoint. This cutoff was chosen because 1) it marks the transition from the moderate to severe stage of the disease and therefore represents a clinically significant landmark and 2) similar outcome scores have 
been previously used., ${ }^{2,32}$ Exploratory analyses of neighboring endpoints (i.e., CMMS $\leq 15$ ) did not change the results.

Functional outcome. Functional capacity was assessed using the Blessed Dementia Rating Scale (BDRS) parts I and II. ${ }^{33}$ The range is between 0 and 17 , with higher scores indicating worse functional status. We chose a BDRS score of $\geq 10 / 17$ as the functional endpoint. The rationale for the functional cutoff was similar to the one described above for the cognitive cutoff. Again, exploratory analyses of neighboring BDRS endpoints gave similar results.

Institutionalization. The "equivalent institutional care" the patient was receiving was rated at each 6-month follow-up interval. This rating is the second section of a Dependency Scale ${ }^{34}$ that rates the patient's need for care. It summarizes the interviewer's impression, based on data from the entire study protocol, of the care the patient receives and requires, regardless of the patient's location. Rating categories are limited home care (independent living, with some help in shopping, cooking, or housekeeping but not with all the tasks); adult home (a supervised setting with regular assistance in shopping, cooking, and housekeeping and constant companionship, security, legal, or financial help); and health-related facility (around-the-clock supervision of personal care, safety, or medical care). We used the equivalent institutional care rating of health related facility as an endpoint for prediction. Interrater reliability for the equivalent institutional care is good, with an intraclass correlation coefficient of $0.73 .^{34}$

Death. We typically learned of patients' deaths via family member contacts or during attempted follow-up. For patients who could not be contacted for Predictors Study follow-up or were otherwise lost to follow-up, death information was obtained as available through the National Death Index. Autopsies were obtained whenever possible.

Statistical analyses. Baseline characteristics of patients who did and those who did not reach the four outcomes of interest during the study period were compared using Student's $t$ test for continuous variables and $\chi^{2}$ test for categorical variables.

We calculated separate Cox proportional hazards models with the following dichotomous outcomes: cognitive endpoint, functional endpoint, institutionalization, and death. Duration (in 6 -month blocks) between the initial visit and either development of the outcome or last evaluation without the outcome served as the timing variable in each above model. In initial models, we considered presence of motor signs at baseline evaluation as a potential predictor. The main analyses considered motor signs, in the form of a time-dependent covariate. In additional Cox models, all five individual motor signs domains (time-dependent) were included in the analyses in a stepwise forward selection mode (entry criterion: $p<0.05$; removal criterion: $p>0.05$ ). The Cox proportional hazards model with time-dependent covariates takes into account changes in the status of the predictor variable at each study visit (e.g., a patient may not have motor signs at the first visit but may manifest motor signs at the second and third visit). In subsequent Cox models, we simultaneously adjusted for the following variables: cohort (first or second Predictors cohort; dichotomous), recruitment center (dummy variable with New York center as the reference), age at intake in the study, sex, education in years, CMMS score at initial evaluation, BDRS score at initial evaluation, and the comorbidity index (dichotomous). Because the ethnic distribution of the patients enrolled in the Predictors study was heavily weighted toward whites (93\%) with very few African Americans $(4.8 \%)$ or Hispanics $(2.7 \%)$, no ethnicity variable was included in the models.

In supplementary analyses we examined the effect of rates of accumulation of motor signs in reaching the outcomes. For patients who had at least three motor sign assessments we calculated slopes of total motor signs continuous-sum scores. We then selected patients with total motor sign score slopes $>0(n=244)$, dichotomized by median slope, and created two groups: one that exhibited faster and one that exhibited slower accumulation of motor signs over time. We then calculated Cox models for all four outcomes including the above dichotomous variable (fast vs slow motor sign accumulation) as the main predictor, controlling for baseline total motor signs score, cohort, recruitment center, age, sex, education, CMMS score at initial evaluation, BDRS score at initial evaluation, and the comorbidity index.
Table 1 Demographic and clinical characteristics of patients

\begin{tabular}{ll} 
Characteristics & Value \\
\hline Cohort 1/Cohort 2, n (\%) & $252(47) / 281(53)$
\end{tabular}

Cohort 1/Cohort 2, n (\%)

$252(47) / 281(53)$

Recruitment center, n (\%)

New York

Baltimore

Boston

Paris

Larissa

Duration of follow-up, y, mean (SD)

Age at study entry, $y$, mean (SD)

$73.9(8.81)$

Education, y, mean (SD)

Men/women, n (\%)

$208(39) / 323(61)$

CMMS at study entry, mean (SD)

$38.2(7.41)$

BDRS at study entry, mean (SD)

Comorbidity Index 0/Comorbidity $358(67) / 175(33)$

Index $\geq 1, \mathrm{n}(\%)$

Main predictors (motor signs), n (\%)

Any motor signs baseline/any evaluation

Speech-facial expression baseline/any evaluation

Tremor baseline/any evaluation

$21(4) / 56(11)$

Rigidity baseline/any evaluation

$30(6) / 131(26)$

Posture-gait baseline/any evaluation

$28(6) / 141(28)$

Bradykinesia baseline/any

$23(5) / 121(24)$ evaluation

Outcomes, n (\%)

Cognitive endpoint during follow-up

199 (39)

Functional endpoint during follow-up

Institutionalized during follow-up

Dead during follow-up

CMMS = Columbia Mini-Mental State Examination; BDRS = Blessed Dementia Rating Scale.

Results. Overall, 533 subjects with $\mathrm{AD}$, approximately half from Predictors 1 and half from Predictors 2 cohort, were included in the study (table 1 ). The majority of patients $(86 \%)$ were recruited from the three centers in the United States. As dictated by the inclusion criteria, patients were at the early stages of $\mathrm{AD}$ at the time of initial recruitment: CMMS was 38.2, corresponding to a MMSE of $\sim 21$. The subjects were, on average, well educated (average 13.1 years of education) and in good general health (as indicated by the fact that $\sim 2 / 3$ had a comorbidity index of 0 ).

Patients were followed for up to 13.1 years, during which time there were 3,149 visit-assessments of motor signs (up to 26 , on average 5.9 per patient). The relatively short average duration of follow-up $(\sim 3$ years) reflects 1$)$ the continuous accrual of subjects even at present and 2 ) that approximately half of the recruited patients have died (median survival from recruitment into the study was 6.3 years [5.7 to 6.9]). During the period each subject was followed, missed visits were rare: $<18 \%$ missed more than one semi-annual visit and $<9 \%$ missed more than two. 
Table 2 Risk ratios (RR) and 95\% CI from Cox models predicting occurrence of the four outcomes

\begin{tabular}{lccc}
\hline $\begin{array}{l}\text { Predictors } \\
\text { dichotomous scores }\end{array}$ & $\begin{array}{c}\text { Cognitive outcome, } \\
\text { RR (95\% CI })\end{array}$ & $\begin{array}{c}\text { Functional outcome, } \\
\text { RR (95\% CI })\end{array}$ & $\begin{array}{c}\text { Institutionalization, } \\
\text { RR (95\% CI })\end{array}$ \\
\hline $\begin{array}{l}\text { Unadjusted models } \\
\text { Any motor signs }\end{array}$ & $1.98(1.46-2.69)$ & $2.55(1.90-3.41)$ & $2.14(1.62-2.84)$ \\
$\begin{array}{l}\text { Adjusted models } \\
\text { Any motor signs }\end{array}$ & $1.72(1.24-2.38)$ & $1.80(1.33-2.45)$ & $1.68(1.26-2.25)$ \\
$\begin{array}{l}\text { Speech-facial expression } \\
\text { Tremor }\end{array}$ & $2.73(1.54-4.85)$ & & $1.38(1.05-1.82)$ \\
$\quad \begin{array}{l}\text { Rigidity } \\
\text { Bradykinesia }\end{array}$ & $1.97(1.30-2.98)$ & $2.42(1.68-3.50)$ & \\
Posture-gait & & & $1.70(1.16-2.49)$ \\
\hline
\end{tabular}

The models of the 1st and 2nd row included any motor signs (in dichotomous form) as time-dependent predictor. In the model shown in the remaining rows all individual domain motor signs (dichotomous, time-dependent) were included in a stepwise forward selection mode (only significant results are tabulated). Adjusted models simultaneously controlled for cohort, recruitment center, age, sex, education, baseline CMMS, baseline BDRS, and comorbidity index.

CMMS = Columbia Mini-Mental State Examination; BDRS = Blessed Dementia Rating Scale.

Follow-up was complete for $94.5 \%$ of the cohort, while only $5.5 \%$ of the cohort ( $\mathrm{n}=27$ subjects) had missing follow-up information for the period of the last year before the most updated data entry. Rates of missing individual motor sign items (when the modified UPDRS scale was administered) ranged between $0.7 \%$ and $4.5 \%$.

At the baseline evaluation, motor signs were present for $14 \%$, while $45 \%$ of the patients developed motor signs at some point during follow-up. Frequencies of individual motor sign domains at baseline were 4 to $6 \%$. As expected, tremor was less frequent: $\sim 25 \%$ of the patients had either speech-facial expression or rigidity or posture-gait or bradykinesia abnormalities, while only $11 \%$ had tremor at some point during follow-up. Details of changes of motor signs during the disease course have been published. ${ }^{4}$ Briefly, total motor signs score increased at an annual rate of $3 \%$ of total possible score. Prevalence of motor signs increased as disease chronicity increased: first year $18 \%$, second year $19 \%$, third year $25 \%$, fourth year $33 \%$, fifth year $46 \%$, sixth year $41 \%$, seventh year $39 \%$, eighth year $47 \%$, ninth year $90 \%$, 10th year $67 \%$, 11th year $62 \%$, 12th year $64 \%$, 13 th year $71 \%$.

Adjusting for cohort, recruitment center, age, sex, education, baseline CMMS, baseline BDRS, and comorbidity index, presence of motor signs in a dichotomous form at initial evaluation was predictive of poor functional outcome (RR: 1.87 [1.24 to 2.82]) and death (RR: 1.66 [1.15 to $2.38]$ ) but not of poor cognitive outcome (RR: 1.37 [0.91 to 2.07]) or institutionalization (RR: 1.41 [0.98 to 2.03]). Similarly, motor sign continuous-sum score at baseline was predictive of poor functional outcome (RR: 1.05 [1.02 to 1.09]) and death (RR: 1.06 [1.03 to 1.09]) but not of poor cognitive outcome (RR: 1.03 [0.99 to 1.07]) or institutionalization (RR: 1.01 [0.98 to 1.04$]$ ).

In survival analyses, presence of time-dependent motor signs was associated with increased risk of reaching all four outcomes in both adjusted and unadjusted models, in either dichotomous or continuous-sum form (tables 2 and 3 ). Overall, $39 \%$ of the patients reached the cognitive endpoint. The associations between motor signs and cognitive outcome were driven primarily by tremor (see tables 2 and
3). The functional endpoint was reached by $41 \%$ of the patients and it was mostly the presence of bradykinesia that predicted poor functional outcome (see tables 2 and 3). Slightly more than half of the patients (54\%) reached the equivalent institutional care endpoint and slightly less than half $(47 \%)$ died during the follow-up period. It was patients with posture-gait impairment that were mostly at risk for institutionalization and death (see tables 2 and 3).

With regard to the effects of the other covariates, the results were as follows (table E-1 on the Neurology Web site at www.neurology.org). As compared to the New York center, risk for institutionalization was higher for the Boston center. Women had higher risk for institutionalization and lower risk for mortality than men. Older patients were less likely to reach the cognitive endpoint but more likely to die. Higher baseline CMMS was associated with reduced risk of reaching all the outcomes. Higher baseline BDRS (indicating worse functional status) was associated with higher risk for functional outcome and institutionalization. Presence of comorbid conditions was associated with higher risk of reaching the institutionalization and death endpoints.

Adjusting for baseline motor sign sum score, cohort, recruitment center, age, sex, education, baseline CMMS, baseline BDRS, and comorbidity index, patients who accumulated motor signs faster had higher risk of reaching the cognitive (RR: 1.58 [1.09 to 2.27]), functional (RR: 1.81 [1.26 to 2.61]), institutionalization (RR: 1.43 [1.03 to 2.00]), and death (RR: 1.69 [1.19 to 2.40]) outcomes.

Discussion. In this study, presence of motor signs during the course of $\mathrm{AD}$ was associated with higher risk for cognitive and functional decline, institutionalization, and death. All these associations were significant even after adjusting for multiple potential confounders. Regarding individual aspects of motor signs, presence of tremor was associated with increased risk for cognitive decline, presence of bradykinesia with increased risk for functional decline, and presence of postural-gait impairments with increased risk for institutionalization and death. 
Table 3 Risk ratios (RR) and 95\% CI from Cox models predicting occurrence of the four outcomes

\begin{tabular}{|c|c|c|c|c|}
\hline Predictors sum scores & $\begin{array}{l}\text { Cognitive outcome, } \\
\text { RR (95\% CI) }\end{array}$ & $\begin{array}{c}\text { Functional outcome, } \\
\text { RR }(95 \% \text { CI })\end{array}$ & $\begin{array}{l}\text { Institutionalization, } \\
\text { RR }(95 \% \mathrm{CI})\end{array}$ & $\begin{array}{c}\text { Death, } \\
\text { RR }(95 \% \mathrm{CI})\end{array}$ \\
\hline \multicolumn{5}{|l|}{ Unadjusted models } \\
\hline Total motor signs sum & $1.07(1.04-1.10)$ & $1.11(1.08-1.13)$ & $1.08(1.05-1.11)$ & $1.02(1.01-1.04)$ \\
\hline \multicolumn{5}{|l|}{ Adjusted models } \\
\hline Total motor signs sum & $1.05(1.02-1.08)$ & $1.08(1.05-1.11)$ & $1.05(1.02-1.07)$ & $1.02(1.00-1.04)$ \\
\hline Speech-facial expression sum & $1.24(1.08-1.42)$ & $1.16(1.01-1.33)$ & & \\
\hline Tremor sum & $1.49(1.17-1.90)$ & & & \\
\hline Rigidity sum & & $1.06(1.00-1.13)$ & & \\
\hline Bradykinesia sum & & $1.24(1.01-1.53)$ & & \\
\hline Posture-gait sum & & & $1.19(1.07-1.32)$ & $1.12(1.04-1.20)$ \\
\hline
\end{tabular}

Faster rate of motor sign accumulation was also associated with higher risk for reaching all outcomes.

Associations of motor signs with functional decline and institutionalization have been noted in previous studies, including those that used the Predictors 1 cohort. $^{35}$ However, the present analyses are much more powerful since we included more than twice as many patients with $\mathrm{AD}$, and data from an additional 6 to 8 years of follow-up. Data from two EU centers were also included, improving the generalizability of the findings. Additionally, we separately examined individual motor sign domains, rates of motor sign accumulation, and controlled for additional covariates, including comorbid diseases.

The noted frequencies of motor signs were within previously reported ranges. ${ }^{1-4}$ Of importance is the notable discrepancy between the frequency of motor signs in the first and all subsequent evaluations. Only $\sim 1 / 3$ of patients who had motor signs at some point during the follow-up had them at first visit. These results reflect the increasing prevalence of these symptoms during the course of disease. ${ }^{3,4}$ Therefore, the usual approach of considering the presence of these symptoms only at baseline could be one of the major explanations for discrepant predictive ability results in the literature. Our own results actually contest this, since motor signs at baseline were significantly associated with some, but not with some other outcomes, while there was a significant relation with all outcomes when the time-dependent version of motor signs was used.

Use of neuroleptic medications in $\mathrm{AD}$ may affect the natural course of the disease, since it has been associated with poor outcomes. ${ }^{36}$ The noted association between motor signs and poor outcomes in this study was not confounded by medication effects. It is possible that motor abnormalities in patients taking neuroleptics are not completely caused by medications but are also partially accounted by $\mathrm{AD}$-related enhanced sensi- tivity to neuroleptics. However, because it is not possible to separate the relative contribution of these two factors and in order to increase our confidence that the occurrence of motor signs in the present study is strictly related to the underlying disease process, we analyze only non-drug induced motor signs.

We used motor signs in both dichotomous and continuous forms. Each approach has its own merits. Use of the continuous form makes better use of the full range of the recorded data and is more powerful. On the other side, using the dichotomous form provides a more practical tool for everyday clinical practice. Close inspection of tables 2 and 3 reveals that use of different forms produces very similar results. For example, in the adjusted models the results were identical for institutionalization and death (posturegait being the only significant predictor in both its dichotomous and continuous form). Similarly, the cognitive outcome was predicted by tremor (in both its dichotomous and continuous form) and the functional outcome by bradykinesia (in both its dichotomous and continuous form). Discrepancies have to do mostly with the metric properties of the measures. We concentrate more in associations deemed significant for both the dichotomous and continuous version of motor signs, in both adjusted and unadjusted models.

In previous studies, tremor has been associated with higher risk for functional decline ${ }^{2}$ and mortality. ${ }^{12}$ In this study we noted associations between tremor and functional decline and tremor and mortality, but the relationships did not reach significance in the adjusted models. Tremor in the present study was mostly associated with higher risk for cognitive decline. In accordance to one study ${ }^{10}$ (but not another ${ }^{2}$ ), we found an association between bradykinesia and poor functional outcomes. As in our study, previous research has found gait disorder to be associated with increased mortality in patients with $\mathrm{PD}^{37}$ and even in nondemented elderly. ${ }^{38}$ Postural and gait 
abnormalities may have placed patients at higher risk for institutionalization and death via increased risk of falling.

Motor signs may reflect higher burden or different type or more biologically detrimental localization of neuropathology. It is conceivable that the association of different aspects of motor signs with different outcomes may be reflecting varying underlying neurotransmitter systems being affected. For example, in $\mathrm{PD}$, tremor and bradykinesia have been viewed as representing more purely dopaminergic manifestations, while posture, balance, and gait disorders may be mediated by other neurotransmitter systems in addition to dopamine..$^{39,40}$ The underlying pathologic substrate of motor signs in $\mathrm{AD}$ is not clear. They may $^{41,42}$ or may not ${ }^{43-47}$ be associated with Lewy bodies. As previously published, ${ }^{4}$ in this study motor signs were not significantly related to Lewy bodies. Extranigral lesions involving mesocortical dopaminergic pathways, ${ }^{9}$ loss of striatal dopamine transporter sites, ${ }^{48}$ and reduced dopaminergic D2 receptors in the putamen ${ }^{49}$ have also been invoked. The dopaminergic system may not be involved in the manifestation of motor signs in $\mathrm{AD}^{10,50}$ or may be involved but via $\mathrm{AD}$ pathology itself. ${ }^{51}$

Our findings with regard to the covariates used in the adjusted models are consistent with previously reported results. Younger age has been associated with more rapid cognitive decline and worse outcomes. ${ }^{52}$ Most previous publications have reported that women with $\mathrm{AD}$ have longer survival than men. ${ }^{15,22,23,53,54}$ Increased risk for institutionalization for women may have to do with cultural issues and traditional sex roles (such as reluctance or inability of husbands to provide home care for their wives). Not surprisingly and similar to other reports, ${ }^{15,22,53}$ we found that presence of comorbid diseases was associated with worse outcomes in $\mathrm{AD}$.

The results of the adjusted models suggest that both motor signs and worse baseline cognitive and functional status are predicting poor outcomes. To the extent that worse cognitive and functional performance indicate higher disease severity, it is not surprising that associations with poor outcomes have been demonstrated almost universally.7,15,20-22,53 The fact that presence and rate of accumulation of motor signs are significant in the adjusted models suggests that motor signs represent a true predictive factor, over and above disease severity measures. However, we cannot completely exclude the possibility that some aspects of disease progression may not be completely captured by the cognitive or functional measures we control for and could potentially be uniquely manifested by the presence of motor signs. To this extent, some of the associations between motor signs and outcome may reflect being further along in the disease course.

This study has limitations. Patients with $\mathrm{AD}$ were selected from tertiary care University hospitals and specialized diagnostic and treatment centers and thus represent a nonrandom sample of those affected by $\mathrm{AD}$ in the population. In addition, the proportion of nonwhites in our sample was small. Therefore our results might not be generalizable to populationbased $\mathrm{AD}$ and all ethnicities. Although we used survival analyses, which take advantage of variable follow-up times, a longer average duration of follow-up may have provided a more complete conclusion. This could have been achieved with enrollment of patients at even earlier stages of their disease or even before symptom onset; however, it is not clear that this would change the results since motor signs are usually absent early in the disease. We did not administer the full UPDRS scale, which may have restricted the full range of impairment, in particular for appendicular bradykinesia. However, the items were selected on the basis of earlier characterization of motor signs in $\mathrm{AD}$. Also, assessment of motor signs is often difficult and rater related. It is also very difficult to distinguish subtleties of various aspects of the motor examination (such as different types of tone, velocity of movements, and gait abnormalities). However, we did not rely on clinical assessments but instead used a scale that has been shown to have good reliability properties and yields high rates of agreements across interviewers. Also, the raters were instructed to rate the parkinsonian aspect of motor abnormalities in patients with $\mathrm{AD}$. Still, it is possible that we had not been able to accurately distinguish between the various qualitative types of motor disturbances.

In general, confidence in our findings is strengthened by several factors. This is one of the largest studies of its kind examining in detail the issue of motor signs in $\mathrm{AD}$, supplying enough power for detection and more precise calculation of effects of interest and ability to control for potential confounders. A major contribution of the present analyses lies in the careful diagnosis and clinical follow-up that patients received. Clinical diagnosis took place in University hospitals with specific expertise in dementia and was based on uniform application of widely accepted criteria via consensus diagnostic conference procedures. The clinical diagnosis of $\mathrm{AD}$ has been confirmed in a high proportion $(96 \%)$ of those who have come to postmortem evaluation. ${ }^{4}$ The patients were followed prospectively, which eliminates the potential biases inherent in deriving information from retrospective chart reviews. Evaluations were performed semiannually, which provides multiple assessments of motor signs and therefore permits more accurate coefficient calculations. They were also considered in a time-dependent fashion. Our study had very high rate of follow-up participation with very few missing data. Clinical signs of interest were ascertained and coded in a standardized fashion at each visit. Most previous reports studied more impaired patients with $\mathrm{AD}$, capturing the disease at its more advanced stages. Baseline CMMS score for this cohort was 38.2, corresponding to a MMSE of $\sim 21$; therefore, patients with $\mathrm{AD}$ were included from early stages so that the co- 
hort describes the full range of progression over time. Patients were drawn from multiple locations, which increases generalizability of the findings. Reported associations between motor signs and outcomes in many previous studies have been confounded by not taking into account use of medications that may produce motor signs. We excluded patient-evaluations with medications that could produce parkinsonism, which provides higher confidence that the occurrence of motor signs in the present study is spontaneous, and related only to the underlying disease processes.

Prognosis is a standard part of a medical evaluation and knowledge of prognostic indicators is important information for practitioners, patients, and families. These data provide a basis for expanding our understanding of predictors in the course of $\mathrm{AD}$. We add to the previous literature by confirming previous reports that motor signs are associated with poor outcomes and by reporting associations between individual domains of motor signs and particular outcomes. The underlying pathophysiologic substrate of the associations between motor signs and clinical outcomes remains to be explored.

\section{References}

1. Ellis RJ, Caligiuri M, Galasko D, Thal LJ. Extrapyramidal motor signs in clinically diagnosed Alzheimer disease. Alzheimer Dis Assoc Disord 1996;10:103-114.

2. Lopez OL, Wisnieski SR, Becker JT, Boller F, DeKosky ST. Extrapyramidal signs in patients with probable Alzheimer disease. Arch Neurol 1997;54:969-975.

3. Wilson RS, Bennett DA, Gilley DW, Beckett LA, Schneider JA, Evans DA. Progression of parkinsonian signs in Alzheimer's disease. Neurology $2000 ; 54: 1284-1289$

4. Scarmeas N, Hadjigeorgiou GM, Papadimitriou A, et al. Motor signs during the course of Alzheimer disease. Neurology 2004;63:975-982.

5. Murman DL, Kuo SB, Powell MC, Colenda CC. The impact of parkinsonism on costs of care in patients with $\mathrm{AD}$ and dementia with Lewy bodies. Neurology 2003;61:944-949.

6. Miller TP, Tinklenberg JR, Brooks JO, 3rd, Yesavage JA. Cognitive decline in patients with Alzheimer disease: differences in patients with and without extrapyramidal signs. Alzheimer Dis Assoc Disord 1991;5 251-256.

7. Kraemer HC, Tinklenberg J, Yesavage JA. 'How far' vs 'how fast' in Alz heimer's disease. The question revisited. Arch Neurol 1994;51:275-279.

8. Chui HC, Lyness SA, Sobel E, Schneider LS. Extrapyramidal signs and psychiatric symptoms predict faster cognitive decline in Alzheimer's disease. Arch Neurol 1994;51:676-681.

9. Morris JC, Drazner M, Fulling K, Grant EA, Goldring J. Clinical and pathological aspects of parkinsonism in Alzheimer's disease. A role for extranigral factors? Arch Neurol 1989;46:651-657.

10. Soininen H, Laulumaa V, Helkala EL, Hartikainen P, Riekkinen PJ. Extrapyramidal signs in Alzheimer's disease: a 3-year follow-up study. J Neural Transm Park Dis Dement Sect 1992;4:107-119.

11. Burns A, Jacoby R, Levy R. Neurological signs in Alzheimer's disease. Age Ageing 1991;20:45-51.

12. Samson WN, van Duijn CM, Hop WC, Hofman A. Clinical features and mortality in patients with early-onset Alzheimer's disease. Eur Neurol 1996;36:103-106

13. Van Dijk PT, Dippel DW, Van Der Meulen JH, Habbema JD. Comorbidity and its effect on mortality in nursing home patients with dementia. J Nerv Ment Dis 1996;184:180-187.

14. Mitchell SL, Rockwood K. The association between parkinsonism, Alzheimer's disease, and mortality: a comprehensive approach. J Am Geriatr Soc 2000;48:422-425.

15. Larson EB, Shadlen MF, Wang L, et al. Survival after initial diagnosis of Alzheimer disease. Ann Intern Med 2004;140:501-509.

16. Haan MN, Jagust WJ, Galasko D, Kaye J. Effect of extrapyramidal signs and Lewy bodies on survival in patients with Alzheimer disease. Arch Neurol 2002;59:588-593.

17. Mortimer JA, Ebbitt B, Jun SP, Finch MD. Predictors of cognitive and functional progression in patients with probable Alzheimer's disease. Neurology 1992;42:1689-1696.
18. Corey-Bloom J, Galasko D, Hofstetter CR, Jackson JE, Thal LJ. Clinical features distinguishing large cohorts with possible $\mathrm{AD}$, probable AD, and mixed dementia. J Am Geriatr Soc 1993;41:31-37.

19. O'Hara R, Thompson JM, Kraemer HC, et al. Which Alzheimer patients are at risk for rapid cognitive decline? J Geriatr Psychiatry Neurol $2002 ; 15: 233-238$

20. Drachman DA, O'Donnell BF, Lew RA, Swearer JM. The prognosis in Alzheimer's disease. 'How far' rather than 'how fast' best predicts the course. Arch Neurol 1990;47:851-856.

21. Schaufele M, Bickel H, Weyerer S. Predictors of mortality among demented elderly in primary care. Int J Geriatr Psychiatry 1999;14:946-956.

22. Moritz DJ, Fox PJ, Luscombe FA, Kraemer HC. Neurological and psychiatric predictors of mortality in patients with Alzheimer disease in California. Arch Neurol 1997;54:878-885.

23. Stern Y, Folstein M, Albert M, et al. Multicenter study of predictors of disease course in Alzheimer disease (the "predictors study"). I. Study design, cohort description, and intersite comparisons. Alzheimer Dis Assoc Disord 1993;7:3-21.

24. Scarmeas N, Brandt J, Albert M, et al. Association between the APOE genotype and psychopathologic symptoms in Alzheimer's disease. Neurology 2002;58:1182-1188.

25. Folstein MF, Folstein SE, McHugh PR. "Mini-mental state". A practical method for grading the cognitive state of patients for the clinician. J Psychiatr Res 1975;12:189-198.

26. Stern Y, Sano M, Paulson J, Mayeux R. Modified mini-mental state examination: validity and reliability. Neurology 1987;37(suppl 1):179.

27. Richards M, Marder K, Bell K, Dooneief G, Mayeux R, Stern Y. Interrater reliability of extrapyramidal signs in a group assessed for dementia. Arch Neurol 1991;48:1147-1149.

28. Bennett DA, Shannon KM, Beckett LA, Goetz CG, Wilson RS. Metric properties of nurses' ratings of parkinsonian signs with a modified Unified Parkinson's Disease Rating Scale. Neurology 1997;49:1580-1587.

29. Richards M, Stern Y, Marder K, Cote L, Mayeux R. Relationships between extrapyramidal signs and cognitive function in a communitydwelling cohort of patients with Parkinson's disease and normal elderly individuals. Ann Neurol 1993;33:267-274.

30. Bennett DA, Shannon KM, Beckett LA, Wilson RS. Dimensionality of parkinsonian signs in aging and Alzheimer's disease. J Gerontol A Biol Sci Med Sci 1999;54:M191-196.

31. Charlson ME, Pompei P, Ales KL, MacKenzie CR. A new method of classifying prognostic comorbidity in longitudinal studies: development and validation. J Chronic Dis 1987;40:373-383.

32. Stern Y, Mayeux R, Sano M, Hauser WA, Bush T. Predictors of disease course in patients with probable Alzheimer's disease. Neurology 1987; 37:1649-1653.

33. Blessed G, Tomlinson BE, Roth M. The association between quantitative measures of dementia and of senile change in the cerebral grey matter of elderly subjects. Br J Psychiatry 1968;114:797-811.

34. Stern Y, Albert SM, Sano M, et al. Assessing patient dependence in Alzheimer's disease. J Gerontol 1994;49:M216-222.

35. Stern Y, Albert M, Brandt J, et al. Utility of extrapyramidal signs and psychosis as predictors of cognitive and functional decline, nursing home admission, and death in Alzheimer's disease: prospective analyses from the Predictors Study. Neurology 1994;44:2300-2307.

36. Lopez OL, Wisniewski SR, Becker JT, Boller F, DeKosky ST. Psychiatric medication and abnormal behavior as predictors of progression in probable Alzheimer disease. Arch Neurol 1999;56:1266-1272.

37. Levy G, Tang MX, Louis ED, et al. The association of incident dementia with mortality in PD. Neurology 2002;59:1708-1713.

38. Wilson RS, Schneider JA, Beckett LA, Evans DA, Bennett DA. Progression of gait disorder and rigidity and risk of death in older persons. Neurology 2002;58:1815-1819.

39. Pillon B, Dubois B, Cusimano G, Bonnet AM, Lhermitte F, Agid Y. Does cognitive impairment in Parkinson's disease result from nondopaminergic lesions? J Neurol Neurosurg Psychiatry 1989;52:201-206.

40. Levy G, Tang MX, Cote LJ, et al. Motor impairment in PD: relationship to incident dementia and age. Neurology 2000;55:539-544.

41. Olichney JM, Galasko D, Salmon DP, et al. Cognitive decline is faster in Lewy body variant than in Alzheimer's disease. Neurology 1998;51: 351-357.

42. Heyman A, Fillenbaum GG, Gearing M, et al. Comparison of Lewy body variant of Alzheimer's disease with pure Alzheimer's disease: Consortium to Establish a Registry for Alzheimer's Disease, Part XIX. Neurology 1999;52:1839-1844

43. McKeith IG, Perry RH, Fairbairn AF, Jabeen S, Perry EK. Operational criteria for senile dementia of Lewy body type (SDLT). Psychol Med 1992;22:911-922.

44. Weiner MF, Risser RC, Cullum CM, et al. Alzheimer's disease and its Lewy body variant: a clinical analysis of postmortem verified cases. Am J Psychiatry 1996;153:1269-1273.

45. Stern Y, Jacobs D, Goldman J, et al. An investigation of clinical correlates of Lewy bodies in autopsy-proven Alzheimer disease. Arch Neurol 2001;58:460-465.

46. Reinikainen KJ, Paljarvi L, Halonen T, et al. Dopaminergic system and 
monoamine oxidase-B activity in Alzheimer's disease. Neurobiol Aging 1988;9:245-252.

47. Snowden MB, Bowen JD, Hughes J, Larson EB. Study of Alzheimer's dementia patients with parkinsonian features. J Geriatr Psychiatry Neurol 1995;8:154-158.

48. Murray AM, Weihmueller FB, Marshall JF, Hurtig HI, Gottleib GL, Joyce JN. Damage to dopamine systems differs between Parkinson's disease and Alzheimer's disease with parkinsonism. Ann Neurol 1995; 37:300-312.

49. Cross AJ, Crow TJ, Ferrier IN, Johnson JA, Markakis D. Striata dopamine receptors in Alzheimer-type dementia. Neurosci Lett 1984; 52:1-6.

50. Tyrrell PJ, Sawle GV, Ibanez V, et al. Clinical and positron emission tomographic studies in the 'extrapyramidal syndrome' of dementia of the Alzheimer type. Arch Neurol 1990;47:1318-1323.

51. Liu Y, Stern Y, Chun MR, Jacobs DM, Yau P, Goldman JE. Pathological correlates of extrapyramidal signs in Alzheimer's disease. Ann Neurol 1997;41:368-374.

52. Ho GJ, Hansen LA, Alford MF, et al. Age at onset is associated with disease severity in Lewy body variant and Alzheimer's disease. Neuroreport 2002;13:1825-1828.

53. Burns A, Lewis G, Jacoby R, Levy R. Factors affecting survival in Alzheimer's disease. Psychol Med 1991;21:363-370.

54. Wolfson C, Wolfson DB, Asgharian M, et al. A reevaluation of the duration of survival after the onset of dementia. N Engl J Med 2001; 344:1111-1116.
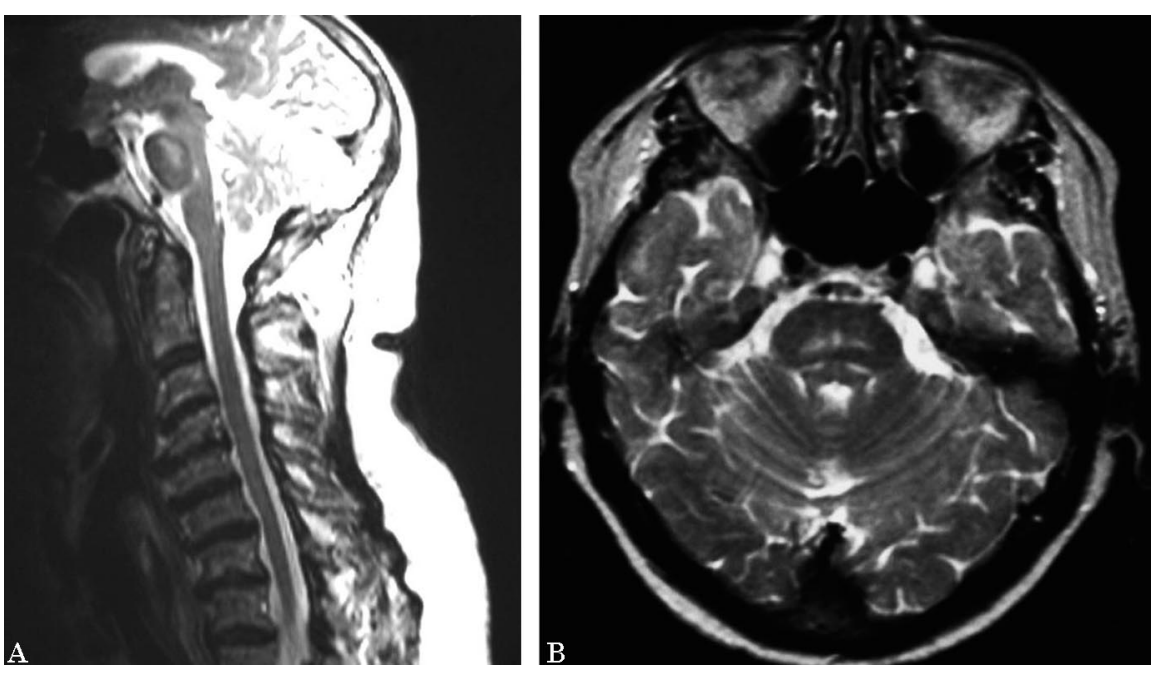

Neurolmages

Figure. Brain MRI, T2-weighted images, sagittal (A) and axial (B) plane, showing a bilateral hyperintense signal in the pons.

\section{Man-in-the-barrel syndrome caused by a pontine lesion}

M. Paulin, MD; J. de Seze, MD; P. Wyremblewski, MD;

H. Zéphir, $M D$; D. Leys, $M D$; and $P$. Vermersch, $M D$, Lille, France

A 78-year-old hypertensive woman suddenly developed an acute brachial diplegia corresponding to man-in-the-barrel (MIB) syndrome. Clinical examination results were otherwise normal, including reflexes. Spinal cord MRI was normal. Brain MRI revealed a bilateral hypersignal in the pons without frontal lesion (figure). Three months later the patient had completely recovered.

Address correspondence and reprint requests to Dr. J. de Seze, Department of Neurology, Hôpital R. Salengro, CHRU de Lille, 59037 Lille Cedex, France; e-mail: j-deseze@chru-lille.fr
MIB syndrome is an unusual feature that has been reported in bilateral watershed infarcts, cervical spinal lesions, and motor neuron disease..$^{1-3}$ The most likely cause in our case was the pontine lesion, presumably of ischemic origin. This is the first case of MIB reported in association with a pontine lesion. It suggests that pyramidal fibers supplying upper-limb function may be located medially at the pontine level.

Copyright $\odot 2005$ by AAN Enterprises, Inc.

1. Crisostomo EA, Suslavich FJ. Man-in-the-barrel syndrome associated with closed head injury. J Neuroimaging 1994;4:116-117.

2. Berg D, Mullges W, Koltzenburg M, Bendszus M, Reiners K. Man-in-thebarrel syndrome caused by cervical spinal cord infarction. Acta Neurol Scand 1998;97:417-419.

3. Katz JS, Wolfe GI, Andersson PB, et al. Brachial amyotrophic diplegia: a slowly progressive motor neuron disorder. Neurology 1999;53:10711076 . 


\title{
Neurology
}

\author{
Man-in-the-barrel syndrome caused by a pontine lesion \\ M. Paulin, J. de Seze, P. Wyremblewski, et al. \\ Neurology 2005;64;1703 \\ DOI 10.1212/01.WNL.0000149910.96498.46
}

This information is current as of May 23, 2005

\section{Updated Information \& Services}

References

Citations

Permissions \& Licensing

Reprints including high resolution figures, can be found at: http://n.neurology.org/content/64/10/1703.full

This article cites 3 articles, 1 of which you can access for free at: http://n.neurology.org/content/64/10/1703.full\#ref-list-1

This article has been cited by 1 HighWire-hosted articles: http://n.neurology.org/content/64/10/1703.full\#\#otherarticles

Information about reproducing this article in parts (figures,tables) or in its entirety can be found online at:

http://www.neurology.org/about/about_the_journal\#permissions

Information about ordering reprints can be found online:

http://n.neurology.org/subscribers/advertise

Neurology ${ }^{\circledR}$ is the official journal of the American Academy of Neurology. Published continuously since 1951, it is now a weekly with 48 issues per year. Copyright . All rights reserved. Print ISSN: 0028-3878. Online ISSN: 1526-632X.

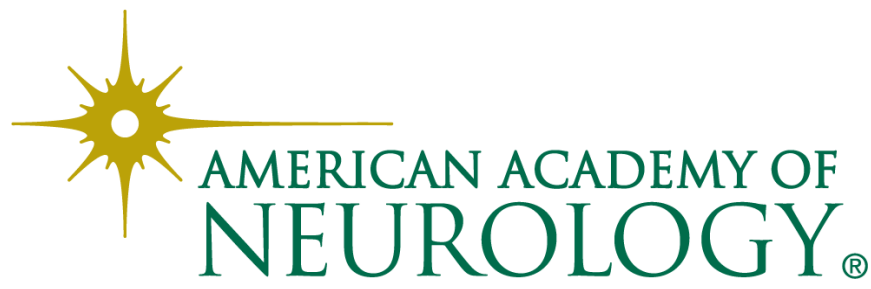

